\title{
Performance of inverter fed HTS induction-synchronous motor operated in liquid nitrogen
}

$\operatorname{AUTHOR}(\mathrm{S})$ :

Nakamura, T; Ogama, Y; Miyake, H

\section{CITATION:}

Nakamura, T ...[et al]. Performance of inverter fed HTS induction-synchronous motor operated in liquid nitrogen. IEEE TRANSACTIONS ON APPLIED SUPERCONDUCTIVITY 2007, 17(2): 1615-1618

\section{ISSUE DATE:}

2007-06

URL:

http://hdl.handle.net/2433/50177

\section{RIGHT:}

(c)2007 IEEE. Personal use of this material is permitted. However, permission to reprint/republish this material for advertising or promotional purposes or for creating new collective works for resale or redistribution to servers or lists, or to reuse any copyrighted component of this work in other works must be obtained from the IEEE. 


\title{
Performance of Inverter Fed HTS Induction-Synchronous Motor Operated in Liquid Nitrogen
}

\author{
Taketsune Nakamura, Yoshio Ogama, and Hironori Miyake
}

\begin{abstract}
We studied the effects of changing the driving of a squirrel-cage type induction motor with high $T_{\mathrm{c}}$ superconducting (HTS) rotor windings. Bi-2223/Ag tape conductors were utilized for the secondary windings and the conventional (normal conducting) stator, 3-phase and 4-pole, was introduced. The fabricated motor was installed in the metal cryostat, and no-load tests were carried out in liquid nitrogen (77 K). A PWM (Pulse Width Modulation) inverter was utilized in order to drive the motor. The test results are discussed based on the nonlinear electrical equivalent circuit. It is shown that the fabricated motor operates not only at slippage mode but also at synchronous mode for the frequency range from $10 \mathrm{~Hz}$ to $60 \mathrm{~Hz}$. Furthermore, the rotating characteristics had frequency dependence, and higher input voltage was necessary for synchronism as the driving frequency increased. These characteristics were explained based on the conventional theoretical discussion and the nonlinear current transport property of HTS windings.
\end{abstract}

Index Terms-Bi-2223/Ag multifilamentary tape, induction motor, liquid nitrogen, PWM inverter, synchronous torque.

\section{INTRODUCTION}

$\mathbf{H}$ IGH $T_{\mathrm{c}}$ superconducting (HTS) motors have widely been developed with the background of advances in high quality HTS tape conductors. Especially, the ship propulsion systems have been considered to be one of the most promising applications for such high performance motors [1], [2]. Although there exist some kinds of wound motors as conventional types, e.g., DC motor, synchronous motor, induction motor, the HTS synchronous one has mostly been developed so far.

On the other hand, HTS induction motor has recently been studied by Sim et al. [3], [4]. They have reported that the synchronous operation is available for such motor, and this is one of the most unique merits compared to the conventional induction motor. Further, we have reported the theoretical analysis based on the nonlinear electrical equivalent circuit [5], and the rotating performance [6]. We call the above-mentioned motor as "HTS induction-synchronous motor," because such motor has both of slippage and synchronous modes.

Manuscript received August 25, 2006. This work was supported in part by the 21st Century COE Program (No.14213201) from The Ministry of Education, Culture, Sports, Science and Technology, Japan.

The authors are with Kyoto University, Kyoto-Daigaku-Katsura 1, Nishikyo-Ku, Kyoto 615-8510, Japan (e-mail: tk_naka@kuee.kyoto-u.ac.jp).

Color versions of one or more of the figures in this paper are available online at http://ieeexplore.ieee.org.

Digital Object Identifier 10.1109/TASC.2007.899896

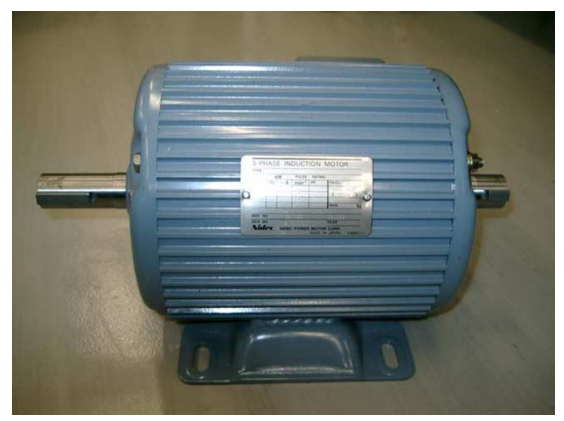

Fig. 1. Overview of subject motor (1.5 kW, $200 \mathrm{~V}, 3$-phase, 4-pole).

For the practical utilization of the motors, the inverter operation is convenient. In this case, the driving frequency dependence of the motor has to be clarified. In this study, we investigate the rotating characteristics of the developed HTS induction-synchronous motor with the use of PWM inverter. The target operating temperature is $77 \mathrm{~K}$. The experimental results are reported and discussed.

\section{EXPERIMENTAL METHOD}

\section{A. Fabrication Details}

Fig. 1 shows the overview of the subject induction motor. The commercialized motor, $1.5 \mathrm{~kW}, 200 \mathrm{~V}, 3$-phase, 4-pole, is considered and only the squirrel-cage windings are replaced with the HTS tapes. For the fabrication of such windings, Bi-2223/Ag multifilamentary tapes are utilized. The specifications of the HTS rotor bars, however, are different from those of the end rings. That is, main limitation of the rotor bar is its size, i.e., the width must be less than $2.5 \mathrm{~mm}$ in order to install the tapes in the rotor slots. Then, the tapes, $2.0 \mathrm{~mm}$ in width, are utilized in this study, and then four of them are bundles for one rotor bar. The critical current of this tape is around 25 A (@77 K and self-field), and the total critical current of one rotor bar is approximately $100 \mathrm{~A}$.

On the other hand, the limitation for the end rings is only its current carrying capability, and the tape, $4.3 \mathrm{~mm}$ in width and $0.22 \mathrm{~mm}$ in thickness, is utilized. We wind this tape 6 turns for one end ring. The critical current of the tape is about $90 \mathrm{~A}$ (@77 K and self-field), and then the total critical current of one HTS end ring is around $540 \mathrm{~A}$. That is, we design that the HTS end rings are always in a dissipationless (zero resistance) state during operation.

The HTS rotor bars and HTS end rings are connected by using solder. Detailed explanation is also found in [6]. 


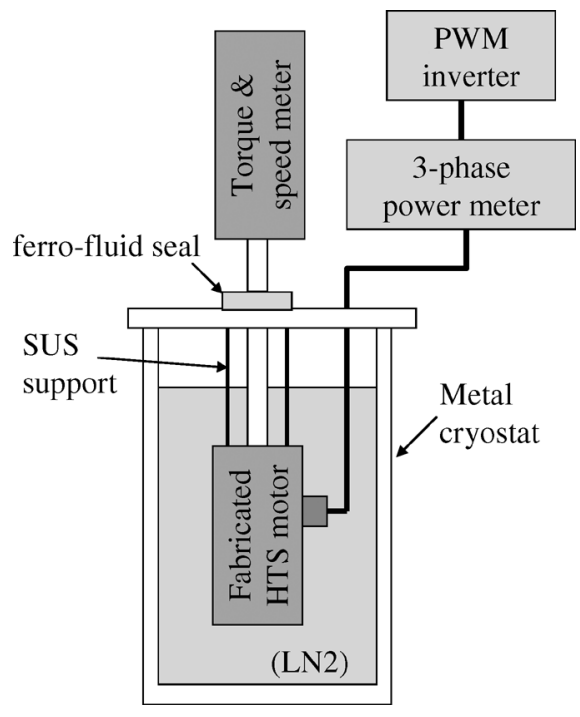

Fig. 2. Schematic diagram of test system.

\section{B. Test Details}

The fabricated HTS induction-synchronous motor is installed in the metal cryostat [6]. It should be noted that the lubrication oil must be removed from the bearing with the use of acetone. This is because such oil will be frozen in a cryogenic temperature. The fabricated motor is hung from the top flange by means of stainless steel support. The torque\&speed meter, which is set in room temperature, is connected with the motor through the ferro-fluid seal (Fig. 2).

After the motor is immersed in liquid nitrogen, the no-load tests are performed for various frequencies at $77 \mathrm{~K}$. The driving frequency is varied by means of typical PWM inverter. The primary information, e.g., voltage, current, power, phase, can be measured by using 3-phase power meter.

\section{ANALYSIS METHOD}

Motor's performance can be analysed based on the electrical equivalent circuit. Fig. 3 shows the commonly utilized electrical equivalent circuit for (a) asynchronous (slippage) mode and (b) synchronous mode, respectively [5], [6]. Where, $V_{1}$ denotes the input phase voltage. And $F^{\prime}\left(I_{2}^{\prime}\right) / s$ and $V_{\mathrm{s}}^{\prime}$ denote the voltage of the secondary (HTS) windings at slip and synchronous operations, respectively. Here, slip, $s$, is defined as $s=\left(N_{\mathrm{s}}-N\right) / N_{\mathrm{s}}$. The parameter $N_{\mathrm{S}}$ means the synchronous speed, and defined with the aids of pole number, $P$, and frequency, $f$, as $120 f / P$, e.g., $1,800 \mathrm{rpm}$ for $f=60 \mathrm{~Hz}$ and $P=4$. The parameters $g_{0}, b_{0}, r$, and $x$, correspondingly, denote the exciting conductance, exciting susceptance, resistance of the windings, and the leakage reactance. Further, the subscript 1 (or 2) shows the primary (or secondary) side, and the prime' shows that the secondary parameter is converted into the primary side. More detailed explanation for the circuits can be found in [5], [6].

\section{RESULTS AND DiscussiON}

\section{A. Rotating Characteristics}

Fig. (4a) shows the experimental results of the no-load speed, $N$, vs. input line voltage, $V_{11}$, curves for different driving fre-

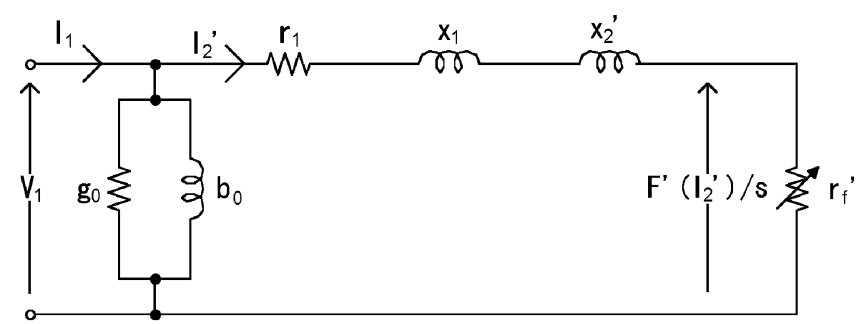

(a)

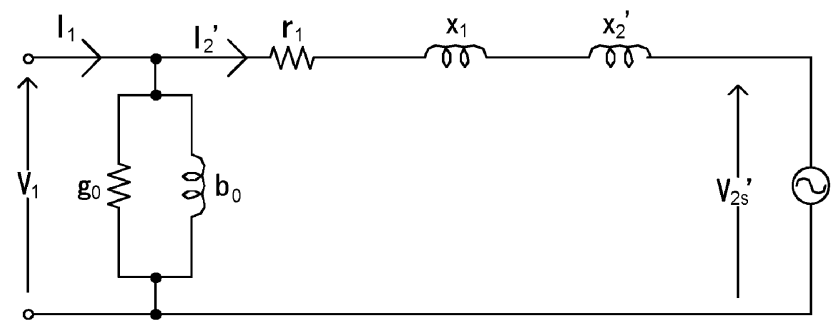

(b)

Fig. 3. Electrical equivalent circuit of HTS induction-synchronous motor for one phase [5], [6]: (a) asynchronous mode; (b) synchronous mode.

quencies at $77 \mathrm{~K}$. As can be clearly seen, the voltage for starting increases by increasing the frequency. We have already proposed that this voltage is called as a minimum starting voltage [5]. The meaning of this voltage can be understood from Fig. (4b), which shows the corresponding primary current, $I_{1}$, vs. $V_{11}$ relations as a fundamental component. The value of $I_{1}$ surely includes the HTS winding's information, and can easily be discussed based on the electrical equivalent circuit as shown in Fig. 3 [5]. The voltages, at which the motor starts, are plotted as empty symbols in the figure. The horizontal broken line also shows the critical current of the HTS rotor bars, $I_{\mathrm{c}}^{\prime}(=3.75 \mathrm{~A})$, that is converted into the primary side of the equivalent circuit. We can see that the starting points are around $I_{\mathrm{c}}^{\prime}$ without frequency dependence, and this means that the motor starts rotating when the rotor bar's induced current overcomes its critical value. In other words, the above-mentioned minimum starting voltage is defined as the voltage at which the rotor bars enter the flux flow (dissipative) state. Therefore, the magnetic fluxes interlink the squirrel-cage windings due to such resistive state, and the starting torque is generated.

As can be seen in Fig. (4a), the motor rotates at slippage mode after starting. Then the rotating speed increases gradually as the voltage is increased. In these asynchronous region, the $I_{1}$ vs. $V_{11}$ curves show almost linear relations except for lower $V_{11}$ region (Fig. (4b)). This linear behavior is because the characteristics are dominated by the leakage reactance rather than the resistance. The gradient of the curve reduces by increasing the driving frequency due to the enlargement of the leakage reactance. It should be noted, however, that the above-mentioned characteristics are not linear when $V_{11}$ is low, and these are because of the effect of the initial magnetization of the iron core.

The current suddenly drops to a small value as the machine reaches synchronism. This phenomenon has already been reported based on the theoretical discussion that the magnetic fluxes are trapped in the dissipationless (zero resistance) secondary windings [5], [6] (compare Figs. (4a) and (b)). In this 


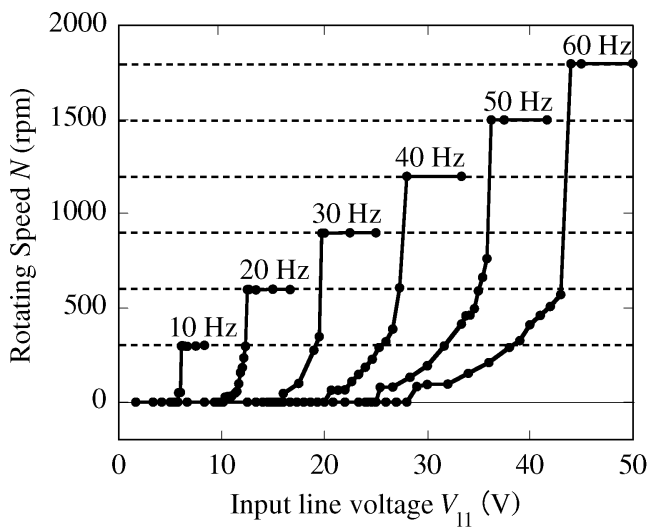

(a)

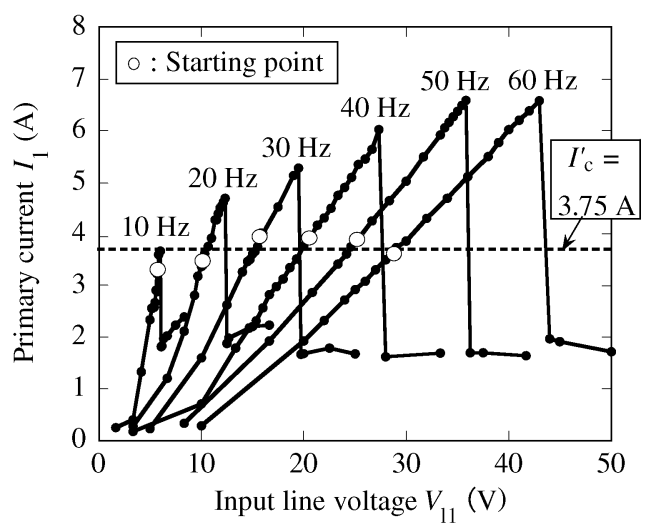

(b)

Fig. 4. Frequency dependence of no-load characteristics at $77 \mathrm{~K}$. The driving frequency is varied by means of PWM inverter: (a) no-load speed, $N$, vs. input line voltage, $V_{11}$; (b) primary current, $I_{1}$, vs. input line voltage, $V_{11}$ as a fundamental component. Empty circles show the starting points of the motor obtained from figure (a).

case, the voltage source is equivalently appeared in the secondary circuit (Fig. (3b)), and then the necessary current for keeping the magnetomotive force decreases. In other words, the fabricated motor behaves like a permanent magnet motor after synchronism. Further, we can see that the primary current is always less than $I_{c}^{\prime}$. Namely, the HTS windings are persistently in the zero resistance state at synchronous rotation. It should be noted that $I_{\mathrm{c}}^{\prime}$ is determined by a typical electric field criterion at $1 \mu \mathrm{Vcm}^{-1}$, and then is not exactly the zero resistance state. It is known that such critical value has little dependence with respect to the frequency in HTS [7]. Therefore, the starting (critical) current of the motor can be estimated by that at DC condition.

\section{B. Frequency Dependency}

Here, we assume that the necessary torque is not dominated by the frequency. In other words, the above-mentioned torque is almost constant without dependence of the frequency. Then, the frequency dependent rotation properties can be explained based on the conventional motor's discussion. If we can assume the relation, $r_{1} \ll x_{1}+x_{2}^{\prime}$, the maximum torque, $\tau_{\mathrm{m}}$, is expressed from Fig. (3a) as follows:

$$
\tau_{\mathrm{m}} \simeq \frac{P}{4 \pi f} \frac{m V_{1}^{2}}{2\left(x_{1}+x_{2}^{\prime}\right)}=\frac{P m}{(4 \pi)^{2}\left(l_{1}+l_{2}^{\prime}\right)}\left(\frac{V_{1}}{f}\right)^{2},
$$

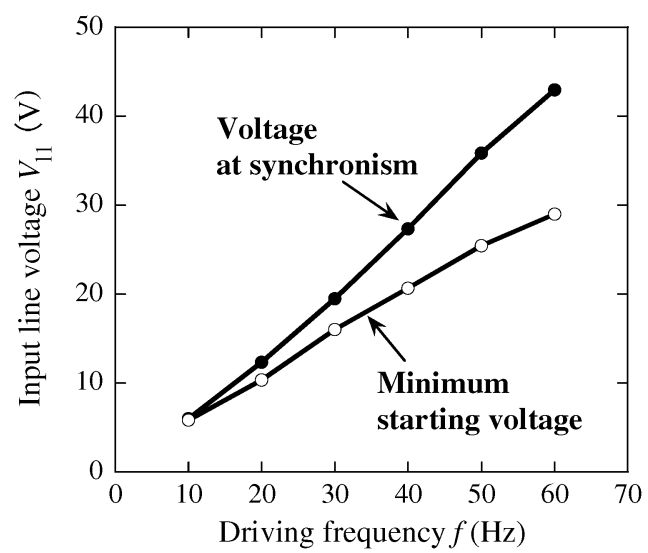

Fig. 5. Input line voltage, $V_{11}$, vs. driving frequency, $f$, relations for starting (empty circles) and pulling-in (solid circles), which is obtained from Fig. (4a).

where, $m(=3)$ and $P(=4)$ are phase number and pole number, respectively. The parameters $l_{1}$ and $l_{2}^{\prime}$ are, correspondingly, the primary and secondary leakage inductances. As can be seen in (1), $\tau_{\mathrm{m}}$ is in proportion to the function of $V_{1} / f$, That is, the voltage for the constant torque becomes smaller by decreasing the driving frequency. As a result, the pull-in voltage becomes smaller correspondingly.

Fig. 5 shows $V_{11}$ vs. $f$ relations for the starting points (empty circles) and the pulling-in points (solid circles), respectively. These plots are obtained from Fig. (4a). We can easily see that both of them show linear relations, at least, from $10 \mathrm{~Hz}$ to $60 \mathrm{~Hz}$. That is, the relation, $V_{1} / f=$ const., is simply valid for the slippage as well as the synchronous operation. This indicates that easier speed control is possible by means of the conventional inverter.

It can also be seen from the figure, on the other hand, that the gradient of the curve at synchronism is steeper than that at the starting. Although, both values are the same at $10 \mathrm{~Hz}$, the voltage range between starting and synchronism, i.e., slippage range, is larger at higher frequencies. In other words, the region of the slippage operation enlarges by frequency. Detailed mechanism of this result must be discussed by considering various reasons, e.g., the nonlinear current transport properties of HTS windings, the effect of the liquid cryogen (liquid nitrogen), etc., and this will be one of our future works.

\section{CONCLUSION}

In this study, frequency dependence of the rotating characteristics in HTS induction-synchronous motor was experimentally investigated by means of the PWM inverter, and discussed based on the electrical equivalent circuits. The fabricated motor was immersed in liquid nitrogen, and then the tests were carried out at $77 \mathrm{~K}$. It was shown that the motor starts rotating when the HTS squirrel-cage windings enter the flux-flow (dissipative) state. Furthermore, so-called minimum starting voltage and pull-in voltage increase by increasing the frequency with the relation, input voltage/driving frequency $=$ const. These behaviors can be explained based on the conventional discussions, and show the possibility of easier operation of the HTS induction-synchronous motor. 


\section{ACKNOWLEDGMENT}

The authors would like to thank Mr. Tsuneo Nakamura for his support of the experimental-setup.

\section{REFERENCES}

[1] H. Sugimoto, T. Tsuda, T. Morishita, Y. Hondou, T. Takeda, H. Togawa, T. Ota, T. Okazaki, and S. Yoshida, "Development of an axial flux inductor type synchronous motor with the liquid nitrogen cooled HTS all windings," in Preprint of Applied Superconductivity Conference 2006, Seattle, USA, 2006, 4LA03.

[2] M. Frank, P. Van Haßelt, P. Kummeth, P. Massek, W. Nick, H. Rothfischer, H. Schmidt, B. Wacker, H.-W. Neumuller, G. Nerowski, J. Frauenhofer, R. Hartig, and W. Rzadki, "High-temperature superconducting rotating machines for ship applications," IEEE Trans. Applied Superconductivity, vol. 16, no. 2, pp. 1465-1468, 2006.
[3] J. Sim, M. Park, H. Lim, G. Cha, J. Ji, and J. Lee, "Test of an induction motor with HTS wire at end ring and bars," IEEE Trans. Applied Superconductivity, vol. 13, no. 2, pp. 2231-2234, 2003.

[4] J. Sim, K. Lee, G. Cha, and J. Lee, "Development of a HTS squirrel cage induction motor with HTS rotor bars," IEEE Trans. Applied Superconductivity, vol. 14, no. 2, pp. 916-919, 2004.

[5] G. Morita, T. Nakamura, and I. Muta, "Theoretical analysis of a YBCO squirrel-cage type induction motor based on an equivalent circuit," $\mathrm{Su}$ perconductor Science and Technology, vol. 19, pp. 473-478, 2006.

[6] T. Nakamura, H. Miyake, Y. Ogama, G. Morita, I. Muta, and T. Hoshino, "Fabrication and characteristics of HTS induction motor by the use of Bi-2223/Ag squirrel-cage rotor," IEEE Trans. Applied Superconductivity, vol. 16, no. 2, pp. 1469-1472, 2006.

[7] T. Nakamura, T. Hoshino, I. Muta, T. Kiss, T. Matsushita, and M. Takeo, "Transport characteristics in YBCO thin films under applying DC and AC currents," in Advances in Superconductivity XI. Tokyo: Springer-Verlag, 1999, pp. 637-640. 